\title{
Strain effect on circularly polarized electroluminescence in transition metal dichalcogenides
}

\author{
Sake Wang $\odot,{ }^{*}$ M. Shoufie Ukhtary $\odot$, and Riichiro Saito $\odot$ \\ Department of Physics, Tohoku University, Sendai 980-8578, Japan
}

(Received 19 June 2020; revised 23 July 2020; accepted 24 July 2020; published 31 August 2020)

\begin{abstract}
Strain effect on circularly polarized electroluminescence (EL) is theoretically analyzed for a p-i-n junction of transition metal dichalcogenides. The electrically controllable circularly polarized EL without the strain effect is understood by valley polarization combined with the electron-hole asymmetry due to the trigonal warping effect in the valence band. The strain enhances circularly polarized EL more efficiently than the trigonal warping effect since the asymmetry occurs in the same direction as the direction of the applied electric field. The calculated result shows that the circular polarization in the strained $\mathrm{MoS}_{2}$ becomes $100 \%$ without losing the EL intensity.
\end{abstract}

DOI: 10.1103/PhysRevResearch.2.033340

\section{INTRODUCTION}

Transition metal dichalcogenide (TMD) has attracted much attention as a two-dimensional (2D) direct-gap semiconductor [1-8]. Since the TMD possesses a broken inversion symmetry [9] at the $K$ and $K^{\prime}$ points in the hexagonal Brillouin zone (BZ), a left- (or right-) handed circularly polarized light (LCP or RCP) is emitted in the $K$ (or $K^{\prime}$ ) valley [10], which is known as the valley polarization [11-13]. If photoemission from the $K$ and $K^{\prime}$ valleys is not equal to each other, we observe a circularly polarized light which is important in optoelectronics [14].

Zhang et al. have fabricated a light-emitting diode (LED) in a p-i-n junction of tungsten diselenide $\left(\mathrm{WSe}_{2}\right)$ [15]. The LED emits circularly polarized electroluminescence (EL) by applying in-plane electric field between the source and drain electrodes. Since the EL photon is emitted in the overlapping region of the occupied $\mathbf{k}$ states by an electron (e) in the conduction band and a hole (h) in the valence band as shown in Fig. 1(a1), the circularly polarized EL occurs when the two overlapping regions in the $K$ and $K^{\prime}$ valleys are not equivalent to each other. This situation occurs when we apply the electric field, E, in the direction of $k_{x}$ [Fig. 1(a2)] in which the overlapping regions change the area since the electron (hole) states shift to left (right) both for the $K$ and $K^{\prime}$ valleys, which breaks the time reversal symmetry between $k_{x}$ and $-k_{x}$ [15]. In order to get the inequivalent area of the two overlapping regions, we need so-called trigonal warping (TW) effect [16-18] in the valence band which we exaggeratedly show as a triangle in Fig. 1. In fact, if the two regions were concentric circles in Fig. 1(a1), the overlapping area in the $K$ and $K^{\prime}$ valleys would be the same even in the presence of the electric field.

\footnotetext{
*Permanent address: College of Science, Jinling Institute of Technology, Nanjing 211169, China; sake@ flex.phys.tohoku.ac.jp

Published by the American Physical Society under the terms of the Creative Commons Attribution 4.0 International license. Further distribution of this work must maintain attribution to the author(s) and the published article's title, journal citation, and DOI.
}

However, since the TW effect is not effective especially for a small circle near the $K\left(K^{\prime}\right)$ point [16-18], we cannot get a large difference of the overlapping area. In this paper, we consider the strain effect on the energy dispersion in which the (e) and (h) circles are originally mutually shifted in the case of $E=0$ as shown in Fig. 1(b1) which has the time-reversal symmetry. When we apply the electric field, the overlapping regions become more inequivalent than the case of the TW effect as shown in Fig. 1(b2), since the direction of the original shift is selected to the direction of the electric field. In the present paper, we show the significant strain effect on circularly polarized EL.

The circularly polarized EL has been observed in other TMDs by similar approach, such as $\mathrm{MoS}_{2}$ [15], $\mathrm{MoSe}_{2}$ [19], and $\mathrm{WS}_{2}$ [20]. Zhang et al. show numerical simulations to explain the degree of circularly polarized EL, $\eta$, [See Eq. (1)] as a function of (1) direction of the in-plane electric field [15], (2) the length of intrinsic (i) region and (3) the voltage between source and drain [21] which is explained by the TW effect. However, it is not clear from the experiment how much the EL intensity (the overlapping area) decreases to get the circularly polarized EL.

In this paper, we investigate a strain effect on the $\eta$ as a function of the strain and electric field. The electronic properties of the strained TMD have been investigated both experimentally $[11,22]$ and theoretically $[23,24]$ in which the monolayer $\mathrm{MoS}_{2}$ can deform up to $11 \%$ [24,25], which is superior to silicon-based devices $[26,27](\sim 1.5 \%$ [28,29]). The flexible property gives an engineering so-called "straintronics" [30]. Yun et $\mathrm{al}$. and other groups show the direct-indirect-gap transition [31-33] and semiconductor-metal transition [34-36] by applying the strain. Rostami et al. [37] theoretically investigates the strain effect on the electronic structure of monolayer TMDs using the effective Hamiltonian [37], which we will adopt to calculate the EL intensity. We show even $\eta=1(100 \%$ circular polarization) without losing the EL intensity, which is much larger than the $\eta$ caused by the TW effect $[38,39]$. Here, the result of TW effect is shown in Sec. S2 of Ref. [40] for comparison.

The paper is organized as follows. In Sec. II, we show the method to calculate the energy band structure and the 


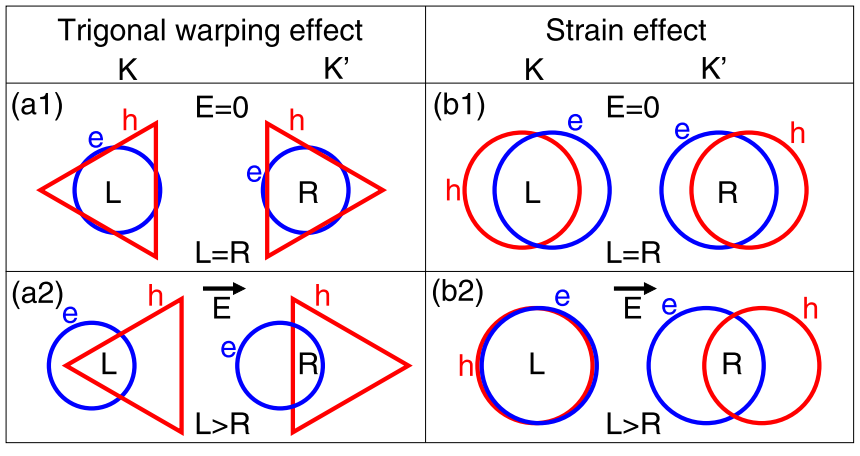

FIG. 1. Occurrence of circularly polarized EL: (a1) the overlapping region of hole (h) and electron (e) occupied regions emits LCP (L) or RCP (R) in the $K$ or $K^{\prime}$ valley, respectively. Here the triangle for $(h)$ represents the trigonal warping effect. When the electric field is zero $E=0$, the two regions have the same area, $\mathrm{L}=\mathrm{R}$. (a2) For $E \neq 0$, (h) [or (e)] shifts to right (left) from which we get $\mathrm{L}>\mathrm{R}$. (b1) When we apply the strain, (h) and (e) are originally shifted in the $K$ and $K^{\prime}$ valleys, which gives $\mathrm{L}=\mathrm{R}$. (b2) For $E \neq 0$, we get $\mathrm{L}>\mathrm{R}$ more effectively than the case of (a2).

asymmetry of the overlapping region. In Sec. III, we show the calculated results of overlapping region as a function of the electric field and strain. By calculating the EL spectra, we evaluate the $\eta$ as a function of the electric field. In Sec. IV, we discuss the parameters that we adapted. Finally, we give the summary in Sec. V.

\section{METHOD}

The degree of circular polarization $\eta$ as a function of the emitted photon energy, $E_{\mathrm{ph}}$, is defined by

$$
\eta\left(E_{\mathrm{ph}}\right)=\frac{I_{+}\left(E_{\mathrm{ph}}\right)-I_{-}\left(E_{\mathrm{ph}}\right)}{I_{+}\left(E_{\mathrm{ph}}\right)+I_{-}\left(E_{\mathrm{ph}}\right)},
$$

where $I_{+}\left(E_{\mathrm{ph}}\right)$ and $I_{-}\left(E_{\mathrm{ph}}\right)$ denote the EL intensity of LCP $(\sigma=1)$ and $\operatorname{RCP}(\sigma=-1)$, respectively. When the $\eta\left(E_{\mathrm{ph}}\right)$ is positive (negative), LCP (RCP) becomes dominant at the $E_{\mathrm{ph}}$. The $I_{+}\left(E_{\mathrm{ph}}\right)$ and $I_{-}\left(E_{\mathrm{ph}}\right)$ in the presence of the in-plane electric field $\mathbf{E}$ is expressed by [41-43]

$$
I_{\sigma}\left(E_{\mathrm{ph}}, \mathbf{E}\right) \propto \sum_{\kappa} \int \frac{1}{\pi} \frac{\gamma\left|M_{\sigma}^{\kappa}(\mathbf{k})\right|^{2} f_{\mathrm{e}}(\mathbf{k}, \mathbf{E}) f_{\mathrm{h}}(\mathbf{k}, \mathbf{E}) d^{2} \mathbf{k}}{\left[\Delta E^{\kappa}(\mathbf{k})-E_{\mathrm{ph}}\right]^{2}+\gamma^{2}},
$$

where $\gamma$ denotes the broadening of the EL spectra due to uncertainty principles for energy. Here we set $\gamma=0.03 \mathrm{eV}$ that is taken from the experimental result [15] of the spectral width of EL. $\Delta E^{\kappa}(\mathbf{k}) \equiv E_{\mathrm{c}}^{\kappa}(\mathbf{k})-E_{\mathrm{v}}^{\kappa}(\mathbf{k})$ denotes the energy difference between the conduction and valence bands at $\mathbf{k}$ in the $K(\kappa=-1)$ or $K^{\prime}(\kappa=1)$ valley. $f_{\mathrm{e}}(\mathbf{k}, \mathbf{E})\left[f_{\mathrm{h}}(\mathbf{k}, \mathbf{E})\right]$ represents the Fermi distribution function of an electron (a hole) in the conduction (valence) band in the quasiequilibrium states for the EL in the presence of $\mathbf{E}$. Using the Boltzmann equation with the relaxation time approximation [44], $f_{\mathrm{e}}(\mathbf{k}, \mathbf{E})\left[f_{\mathrm{h}}(\mathbf{k}, \mathbf{E})\right]$ is given by $[45,46]$

$$
f_{\mathrm{e}, \mathrm{h}}(\mathbf{k}, \mathbf{E}) \approx f_{\mathrm{e}, \mathrm{h}}^{0}\left(\mathbf{k} \pm \frac{e}{\hbar} \tau \mathbf{E}\right)
$$
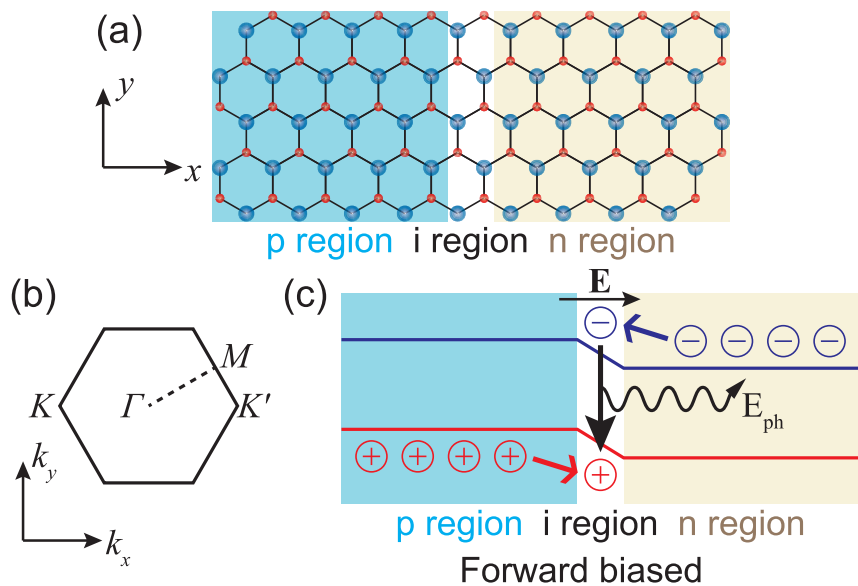

FIG. 2. (a) A TMD p-i-n junction consists of "p", "i", and "n" regions from left to right. In the top view, a metal (blue) and two overlapping chalcogen atoms (red) are seen. (b) The 2D BZ with high-symmetry points, $\Gamma, M, K$, and $K^{\prime}$. (c) Band diagram of the junction by applying the forward bias. In the "i" region, the in-plane electric field $\mathbf{E}$ appears in the $x$ direction. The electrons and holes are injected by $\mathbf{E}$ into the i region where they recombine to give EL.

where the \pm sign accounts for an electron $(+)$ and a hole $(-) . f_{\mathrm{e}, \mathrm{h}}^{0}(\mathbf{k})$ denotes the Fermi distribution function in the equilibrium. Here $\tau$ and $e>0$ denote, respectively, the relaxation time and the elementary charge. We assume that the $\tau$ 's for the electron and the hole are identical in a monolayer TMD [15,47]. Square of the matrix element $\left|M_{\sigma}^{\kappa}(\mathbf{k})\right|^{2}$ in Eq. (2) which defines the photoemission probability of LCP or RCP per unit time at $\mathbf{k}$, is calculated within the dipole approximation $[13,48]$ as follows [40]:

$$
\left|M_{\sigma}^{\kappa}(\mathbf{k})\right|^{2} \propto \frac{27}{8}\left|C_{\mathrm{c}}^{\kappa *}(\mathbf{k}) C_{\mathrm{v}}^{\kappa}(\mathbf{k})\right|^{2} m_{\mathrm{opt}}^{2}(1-\sigma \kappa),
$$

where $C_{\mathrm{c}}^{\kappa}(\mathbf{k})$ and $C_{\mathrm{v}}^{\kappa}(\mathbf{k})$ are the coefficients of the wavefunctions of conduction and valence bands, respectively, which are obtained by solving the effective Hamiltonian as shown below. $m_{\mathrm{opt}}$ denotes atomic optical matrix element which is defined by $m_{\mathrm{opt}}=\left\langle d_{z^{2}}\left(\mathbf{r}-\mathbf{R}_{1}\right)\left|\frac{\partial}{\partial x}\right| d_{x^{2}-y^{2}}(\mathbf{r})\right\rangle-$ $\left\langle d_{z^{2}}\left(\mathbf{r}-\mathbf{R}_{1}\right)\left|\frac{\partial}{\partial y}\right| d_{x y}(\mathbf{r})\right\rangle$, where $\mathbf{R}_{1}$ is a primitive vector in the $x$ direction [40]. The factor $(1-\sigma \kappa)$ in Eq. (4) means that the valley polarization occurs in which LCP $(\sigma=1)$ [or RCP $(\sigma=-1)]$ is emitted only in the $K(\kappa=-1)$ [or $\left.K^{\prime}(\kappa=1)\right]$ valley.

In Fig. 2(a), we illustrate a p-i-n junction made by TMD. The $p-i-n$ junction consists of "p", "i", and " $n$ " regions from the left to right. In the experiment, the $\mathrm{p}$ and $\mathrm{n}$ regions are formed by electrochemical doping of a hole and electron, respectively, by applying a gate voltage $[15,49]$. For simplicity, we assume that the doping is not further affected by the in-plane electric field E. In Fig. 2(b), we show the 2D BZ of the TMD with the high-symmetry points $\Gamma, M, K$, and $K^{\prime}$. In Fig. 2(c), we show a band diagram in which an intrinsic electric field exists in the $\mathrm{i}$ region that are made by the deformation of energy bands so that we can have the Fermi energy of the junction. When we apply a forward bias, a hole (an electron) is injected from the $\mathrm{p}(\mathrm{n})$ region to the $\mathrm{i}$ region in which a pair of an electron and a hole at $\mathbf{k}$ recombine to emit a 
photon with energy $E_{\mathrm{ph}}=\Delta E^{\kappa}(\mathbf{k})$. Such EL occurs at $\mathbf{k},(1)$ if an electron exists in the conduction band, and (2) if a hole exists in the valence band, whose probability is expressed by the product $f_{\mathrm{e}}(\mathbf{k}, \mathbf{E}) f_{\mathrm{h}}(\mathbf{k}, \mathbf{E})$ in Eq. (2).

Using the geometry of Fig. 2(a), let us define in-plane strain in the device. Defining the displacement vector $\mathbf{u}=\left(u_{x}, u_{y}\right)$, the strain tensor is given by $[50,51]$

$$
\varepsilon=\left(\begin{array}{cc}
\varepsilon_{x} & 0 \\
0 & \varepsilon_{y}
\end{array}\right)
$$

where $\varepsilon_{x}=\frac{\partial u_{x}}{\partial x}$ and $\varepsilon_{y}=\frac{\partial u_{y}}{\partial y}$ are strain in $x$ and $y$ directions, respectively. Johari et al. [35] and Kumar et al. [52] pointed out from the DFT calculations that the bottom of conduction band and the top of valence band are found to remain around the $K$ point by expanding and compressing the monolayer $\mathrm{MoS}_{2}$ in the $x$ and $y$ directions, up to $10 \%$, respectively. Thus we set a fixed ratio of the strain in the present calculation: $0 \leqslant \varepsilon_{x}=-\varepsilon_{y} \equiv \varepsilon_{0} \leqslant 0.1$.

In order to obtain $C_{\mathrm{c}}^{\kappa}(\mathbf{k}), C_{\mathrm{v}}^{\kappa}(\mathbf{k})$, and $m_{\mathrm{opt}}$ in Eq. (4), we adopt a spinless effective Hamiltonian given by Rostami et al. [Eq. (7) in Ref. 37] which reproduces the energy dispersion of the strained TMD. The $2 \times 2$ effective Hamiltonian can be described by the identity matrix $s_{0}$ and the Pauli matrices, $s_{x}$, $s_{y}$ and $s_{z}$ [53], as follows:

$$
H=h_{0} s_{0}+h_{1} s_{x}+h_{2} s_{y}+h_{3} s_{z}=\left(\begin{array}{cc}
h_{0}+h_{3} & h_{1}-i h_{2} \\
h_{1}+i h_{2} & h_{0}-h_{3}
\end{array}\right),
$$

where $h_{0}, h_{1}, h_{2}$, and $h_{3}$ are functions of the two-dimensional wavevector $\mathbf{k}=\left(k_{x}, k_{y}\right)$ measured from the $K(\kappa=-1)$ and $K^{\prime}(\kappa=1)$ points, which are defined by

$$
\begin{aligned}
& h_{0}=\frac{S_{+}+S_{-}}{2}+\frac{\hbar^{2} \alpha}{4 m_{0}}\left[\left(k_{x}+\kappa A_{2}\right)^{2}+k_{y}^{2}\right], \\
& h_{1}=t_{0} a_{0}\left(\kappa k_{x}+A_{1}\right), \quad h_{2}=t_{0} a_{0} k_{y}, \text { and } \\
& h_{3}=\frac{\Delta+S_{+}-S_{-}}{2}+\frac{\hbar^{2} \beta}{4 m_{0}}\left[\left(k_{x}+\kappa A_{3}\right)^{2}+k_{y}^{2}\right] .
\end{aligned}
$$

Here, $m_{0}$ is the mass of an electron and $\Delta$ represents the bandgap of the unstrained TMD. $\alpha$ and $\beta$ are dimensionless parameters to reproduce the strain effect in TMD. $S_{ \pm} \equiv 4 \varsigma_{ \pm} \varepsilon_{0}^{2}$ are strain-dependent scalar potentials, in which $\varsigma_{ \pm}$are coefficients of the strain for the lowest conduction band $(+)$ and for the highest valence band $(-)$. In the presence of the strain, $k_{x}$ in the $\kappa$ valley is shifted by the pseudovector potential defined by $A_{i}=\frac{2 g_{i}}{a_{0}} \varepsilon_{0},(i=1,2,3)$ in which $g_{i}$ is the dimensionless parameters and $a_{0}$ is the metal-chalcogenide bond length projected on the $x y$ plane [37]. The $A_{1}$ term has been frequently discussed in graphene [24,54-61]. The $A_{2}$ and $A_{3}$ terms are newly introduced in the strained TMD [62]. It is important to note that in the zigzag nanoribbon as shown in Fig. 2(a), the shift in $k_{y}$ does not appear since the pseudovector potential for $k_{y}, A_{y}$, does not exist in Eq. (7). It is because that the $A_{y}$ is proportional to difference of the bond lengths for the two symmetric nearest-neighbor bonds to the $y$ axis [see Eq. (31) in Ref. 55] and that the difference does not occur for the present strain $\varepsilon_{x}=-\varepsilon_{y} \equiv \varepsilon_{0}$. Thus the energy band does not shift in the $k_{y}$ direction but only in the $k_{x}$ direction when the strain is applied.
For the monolayer $\mathrm{MoS}_{2}$, the dimensionless parameters, $g_{i}(i=1,2,3), \alpha$, and $\beta$ are fitted to $g_{1}=0.002, g_{2}=$ $-56.551, g_{3}=1.635, \alpha=-0.01$, and $\beta=-1.54$; while the other energy parameters are given by $\varsigma_{+}=15.99 \mathrm{eV}, \varsigma_{-}=$ $15.92 \mathrm{eV}, \Delta=1.82 \mathrm{eV}$, and $t_{0}=2.34 \mathrm{eV}$ [37]. According to the DFT result [35], even for $\varepsilon_{0}=0.1$, the lattice constants of the strained TMD change within $5 \%$ of the unstrained ones. This is the reason why we assume that $a_{0}=\frac{a}{\sqrt{3}}$ is a constant for all $\varepsilon_{0}$, where the lattice constant $a$ for unstrained $\mathrm{MoS}_{2}$ is given by $a=3.18 \AA$ [35].

By solving the effective Hamiltonian in Eq. (6), we get the energy dispersion of the conduction $(+)$ and the valence $(-)$ bands as a function of $\mathbf{k}$ and $\varepsilon_{0}$ in the $K\left(K^{\prime}\right)$ valley as follows:

$$
E_{\mathrm{c}, \mathrm{v}}^{\kappa}\left(\mathbf{k}, \varepsilon_{0}\right)=h_{0} \pm \sqrt{h_{1}^{2}+h_{2}^{2}+h_{3}^{2}} \equiv h_{0} \pm \sqrt{G} .
$$

The corresponding wave functions are given by

$$
\mathbf{C}_{\mathrm{c}, \mathrm{v}}^{\kappa}\left(\mathbf{k}, \varepsilon_{0}\right)=\frac{1}{\sqrt{2 G \mp 2 h_{3} \sqrt{G}}}\left(\begin{array}{c}
h_{1}-i h_{2} \\
\pm \sqrt{G}-h_{3}
\end{array}\right) .
$$

It is important to point out that since the $h_{0}$ is a function of $\mathbf{k}$, the energy dispersion of the conduction and valence bands are not symmetric in energy. On the other hand, if the $h_{0}$ is a constant, the two energy bands would be symmetric. This gives the different shifts of the energy dispersion of the conduction and the valence bands in the $\mathbf{k}$ as shown in Fig. 1(b1).

\section{CALCULATED RESULTS OF EL}

In Fig. 3(a), we plot the energy dispersion of $\mathrm{MoS}_{2}$ for the conduction (up) and valence (down) bands on the $k_{x}$ axis near the $K$ (left) and $K^{\prime}$ (right) points in the presence of the strain $\left(\varepsilon_{x}=-\varepsilon_{y}=\varepsilon_{0}=0.05\right)$. As shown in Fig. 3(a), the strained TMD shows an indirect gap both for the $K$ and $K^{\prime}$ valleys. In Fig. 3(b), we plot equienergy contours for the pseudo Fermi energy $\left(E_{\mathrm{F}}=0.02 \mathrm{eV}\right)$ for the electron (blue loop) measured from the bottom of the conduction band and the hole (red loop) from the top of the valence band. Here, the pseudo Fermi energy is defined by the maximum energy of the electrons or the holes in which the electrons or the holes are, respectively, injected in the conduction or the valence band in the $i$ region. Since the inside of the red (blue) loop corresponds to the occupied states by the electrons (the holes), the EL occurs in the overlapping regions $\left[f_{\mathrm{e}}(\mathbf{k}, \mathbf{E}) f_{\mathrm{h}}(\mathbf{k}, \mathbf{E}) \neq 0\right]$ of the two loops. It is noted that the relative portion of the overlapping area to the loop area decreases with increasing $E_{\mathrm{F}}$ for a given value of the shift.

The relative shift of the two loops are symmetric for the $K$ and $K^{\prime}$ valleys since the pseudovector potential does not break the time-reversal symmetry. The relative shift at each valley occurs by the strain effect. In fact, the shift does not occur when we select $\varepsilon_{0}=0$ in the Hamiltonian [Eq. (6)]. When we discuss the strain effect, since the relative shift made by the strain is more effective than that by the TW effect even for a small $\varepsilon_{0}=0.01$, thus we neglect the TW effect in the present Hamiltonian.

In Fig. 4, we plot $\Delta k_{x}^{\mathrm{m}}$ as a function of $\varepsilon_{0}$, which is the difference of the $k_{x}$ between the bottom of the conduction band and the top of the valence bands as shown in Fig. 3(b). 

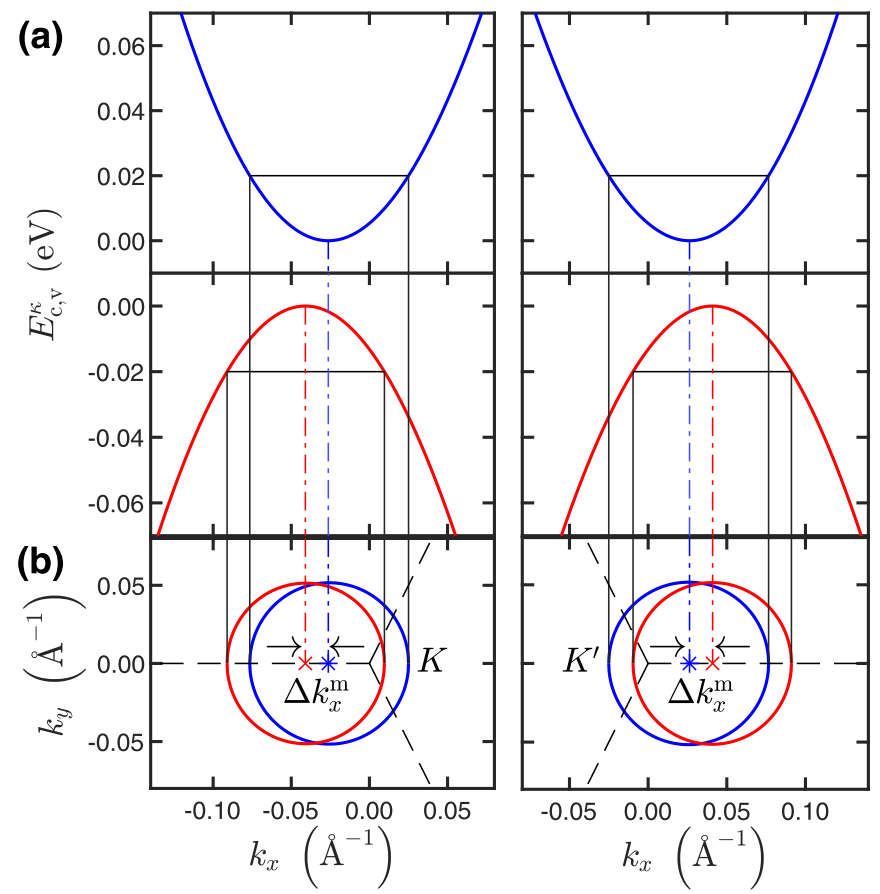

FIG. 3. (a) Energy dispersion of $\mathrm{MoS}_{2}$ for the conduction band (up) and the valence band (down) on the $k_{x}$ axis near the $K$ (left) and $K^{\prime}$ (right) point in the presence of the strain $\left(\varepsilon_{x}=-\varepsilon_{y}=\varepsilon_{0}=0.05\right)$. The strained TMD has an indirect gap. (b) Equienergy contours of the electron (blue loop) and the hole (red loop) distributions in the k-space in the $K$ (left) and $K^{\prime}$ (right) valleys. Here we assume the pseudo Fermi energy for the electrons and the holes are taken to be $E_{\mathrm{F}}=0.02 \mathrm{eV}$.

We also plot the value of the indirect gap $E_{\mathrm{g}}$ as a function of $\varepsilon_{0}$ in Fig. 4. The $\Delta k_{x}^{\mathrm{m}}\left(E_{\mathrm{g}}\right)$, which occurs by the k-dependent $h_{0}$, monotonically increases (decreases) with increasing $\varepsilon_{0}$ which reproduces the DFT result well $[35,52]$.

When we apply the in-plane electric field $\mathbf{E}$ in the i region, the occupied $\mathbf{k}$ region of the electrons (or holes) shifts from the equi-enegy loop to a shifted loop of $\mathbf{k} \mp \frac{e}{\hbar} \tau \mathbf{E}$. This means that the charge occupation of electrons (or holes) is shifted

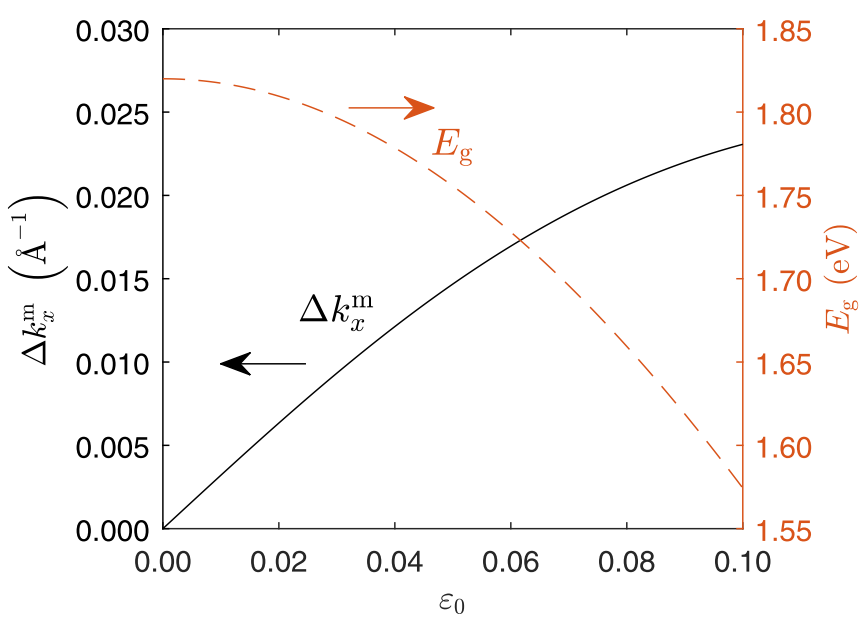

FIG. 4. The difference of the $k_{x}$ and energy gap, $\Delta k_{x}^{\mathrm{m}}$ and $E_{\mathrm{g}}$, respectively, between the bottom of the conduction band and the top of the valence band at the $K$ valley are plotted as a function of $\varepsilon_{0}$.
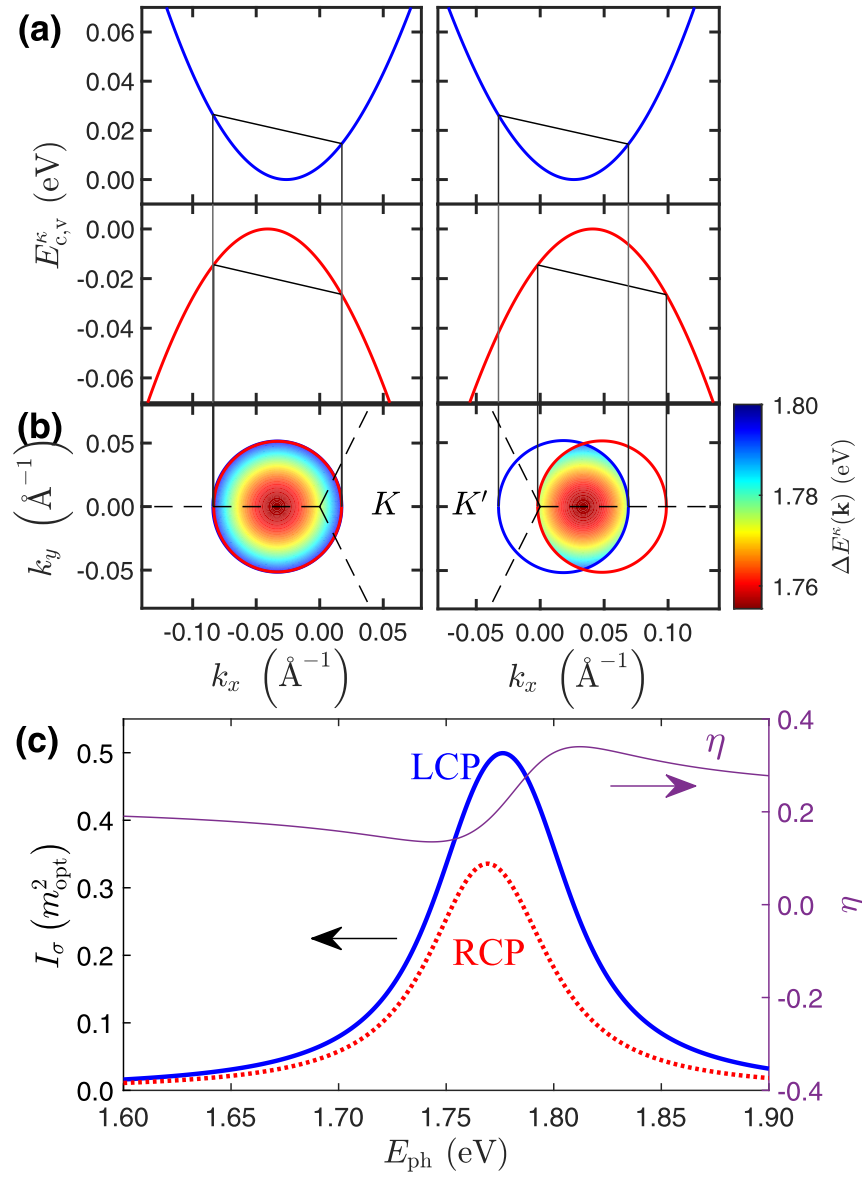

FIG. 5. (a) In the presence of $\mathbf{E}$, the occupied states of electrons (top) and holes (bottom) are shifted in the opposite direction to each other. (b) The shifted loops of electrons (blue line) and holes (red line) are plotted in the $\mathbf{k}$-space. In the overlapping region, the color represents $\Delta E^{\kappa}(\mathbf{k})=E_{\mathrm{c}}^{\kappa}(\mathbf{k})-E_{\mathrm{v}}^{\kappa}(\mathbf{k})=E_{\mathrm{ph}}$ with the corresponding color scale. (c) The EL spectra of LCP $I_{+}$and RCP $I_{-}$(left) and the degree of circular polarization $\eta$ (right) as a function of $E_{\mathrm{ph}}$. We set $\tau E=5 \times 10^{-8} \mathrm{~N} \mathrm{~s} \mathrm{C}^{-1}$. Other parameters are the same as those in Fig. 3 .

in the fixed energy dispersion in the presence of $\mathbf{E}$ as shown in Fig. 5(a). This situation is similar to that water surface in a PET bottle is tilted under acceleration. Since the loops of electron and hole are shifted in opposite directions to each other for a given $\mathbf{E}$ and since the loops of electron (or hole) are shifted in the same direction for the $K$ and $K^{\prime}$ valleys, the overlapping regions in the $K$ and $K^{\prime}$ valleys become inequivalent, as shown in Fig. 5(b), which is the origin of circularly polarized EL emission in the strained TMD.

In Fig. 5(a), we illustrate the tilted occupied $\mathbf{k}$ states of electrons and holes in the presence of $\mathbf{E}$ along the $k_{x}$ direction. In the plot, we adopt $\tau E=5 \times 10^{-8} \mathrm{~N} \mathrm{~s} \mathrm{C}^{-1}$ and the other parameters are taken to be the same as those in Fig. 3. In Fig. 5(b), we plot the shifted loops of electrons (red loops) and holes (blue loops) in the $K$ (left) and $K^{\prime}$ (right) valleys. As seen in Fig. 5(b), the overlapping regions are colored in which the color represents the energy difference between the conduction and valence bands $\Delta E^{\kappa}(\mathbf{k})$. The corresponding colorbar is shown to the right. Since the value of $\Delta E^{\kappa}(\mathbf{k})$ 
corresponds to the photon energy of EL, $E_{\mathrm{ph}}$, we can calculate the EL spectra by integrate on $\mathbf{k}$ using Eq. (2) and we get the $\eta$ by Eq. (1) as shown in Fig. 5(c). Here we assume that the optical matrix element $m_{\mathrm{opt}}$ is a constant and does not depend on the strain nor the electric field for simplicity.

In Fig. 5(c), we plot the EL spectra of LCP $I_{+}$from the $K$ valley and RCP $I_{-}$from the $K^{\prime}$ valley and the $\eta$ as a function of $E_{\mathrm{ph}}$. It is clear from Fig. 5(b), since the overlapping area for LCP $(K)$ is larger than that for RCP $\left(K^{\prime}\right)$, the EL of LCP always has a larger intensity than that of RCP which gives a much larger $\eta \sim 0.2$ than that for the TW effect $(\eta \sim 0$ shown in Fig. S6 [40]). For a positive $E$, the two loops shifted by the strain are almost overlapped because of the shift by the electric field in the $K$ valley, while the loops in the $K^{\prime}$ valley are separated by the electric field. Thus it is clear that by changing the sign of $E$, we can get the negative value of the $\eta$.

It is noted here that the peak position of $E_{\mathrm{ph}}$ for the EL of LCP is higher than that of RCP. It is because that a larger overlapping area generally gives a larger $\Delta E^{\kappa}(\mathbf{k})$ as can be seen from the colormap in Fig. 5(b). Thus the $\eta$ becomes larger in a higher energy region of $E_{\mathrm{ph}}>1.79 \mathrm{eV}$ than $E_{\mathrm{ph}}<$ $1.77 \mathrm{eV}$ which is also understood by the blueshift in EL spectra of LCP.

When we increase the value of $\tau E$, an extreme case of EL with $100 \%$ LCP $(\eta=1)$ occurs as shown in Fig. 6 in which we adopt $\tau E=2.8 \times 10^{-7} \mathrm{~N} \mathrm{~s} \mathrm{C}^{-1}$. In Fig. 6(a), we show the occupation of electrons and holes in the conduction (top) and valence (bottom) bands, respectively, for the $K$ (left) and $K^{\prime}$ (right) valleys. In this case, as shown in Fig. 6(b), the overlapping region does not exist in the $K^{\prime}$ valley while the overlapping region survives in the $K$ valley. Thus we get only EL intensity of LCP from the $K$ valley and $\eta=1$ as shown in Fig. 6(c). It should be mentioned that the EL intensity of LCP in Fig. 6(c) becomes much smaller than that in Fig. 5(c) because of the reduction (20\%) of the overlapping area. For the TW effect shown in Fig. S5 [40] with equivalent parameters, we only have $\eta \sim 0.006$.

In order to get a large $\eta$ without losing the EL intensity, we need (1) a large $E_{\mathrm{F}}$ for a large area of a loop, (2) a large strain $\varepsilon_{0}$ for a large original shift of the two loops and (3) a large $\tau E$ for making inequivalent overlapping areas in the $K$ and $K^{\prime}$ valleys. Another important fact is that the relative shift in the EL-spectral peak of LCP and RCP can give a large $\eta$.

Since the in-plane electric field is the key factor for generating the circularly polarized EL, let us discuss the effect of $\tau \mathbf{E}$ on $\eta$. In Fig. 7(a), we plot the photon energy at the maximum EL intensity $E_{\mathrm{ph}, \sigma}^{\mathrm{m}}$ for LCP and RCP as a function of $\tau E$ from the negative to positive values. Here $\tau E<0$ means that we exchange the $\mathrm{p}$ and $\mathrm{n}$ region so that we can keep the forward bias condition with exchanging the direction of $\mathbf{E}$. As shown in Fig. 7(a), there is a maximum of $E_{\mathrm{ph}, \sigma}^{\mathrm{m}}$ for LCP (RCP) at a value denoted by $\tau E_{\mathrm{cr}}^{+}\left(\tau E_{\mathrm{cr}}^{-}\right)$. At the $\tau E_{\mathrm{cr}}^{+}\left(\tau E_{\mathrm{cr}}^{-}\right)$, the EL intensity of LCP (RCP) becomes maximum, too, as is shown in Fig. 7(b), in which we plot the maximum intensity $I_{\sigma}^{\mathrm{m}}$ (left) in unit of $m_{\mathrm{opt}}^{2}$ and $\eta$ (right) at $E_{\mathrm{ph}}^{\mathrm{m}}$ as a function of $\tau E$, where $E_{\mathrm{ph}}^{\mathrm{m}}$ is defined by $\frac{1}{2} \sum_{\sigma} E_{\mathrm{ph}, \sigma}^{\mathrm{m}}$.

Let us explain the reason of the behavior shown in Fig. 7 for a positive $\tau E$. When $E$ increases from zero to the critical electric field $E_{\mathrm{cr}}^{+}$, the loops of electrons and holes move to
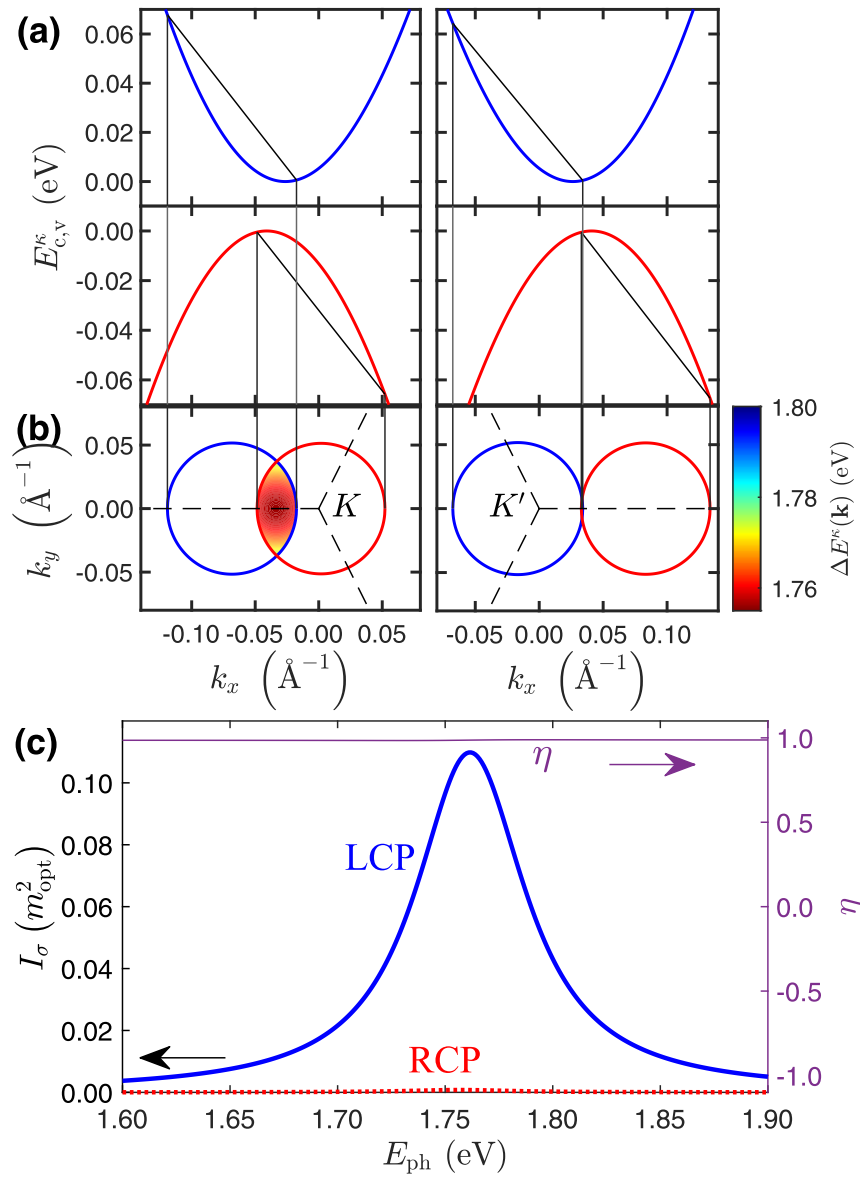

FIG. 6. (a) An extreme case for the tilt of the occupied $\mathbf{k}$ states of electrons and holes in the presence of $\mathbf{E}$. (b) The loops of occupied states for electrons (blue line) and holes (red line) in the $\mathbf{k}$-space. Here we set $\tau E=2.8 \times 10^{-7} \mathrm{~N} \mathrm{~s} \mathrm{C}^{-1}$. Other parameters are the same as those in Fig. 5. (c) The EL spectra of LCP $I_{+}$and RCP $I_{-}$ (left) and the degree of circular polarization $\eta$ (right) as a function of $E_{\text {ph }}$. Only the LCP emission from the $K$ valley occurs because of the vanishing overlapping region in the $K^{\prime}$ valley.

increase (decrease) the overlapping area in the $K\left(K^{\prime}\right)$ valley. Therefore $I_{+}^{\mathrm{m}}\left(I_{-}^{\mathrm{m}}\right)$ increases (decreases) with the blueshift (redshift) of LCP (RCP). The value of $E_{\text {cr }}^{+}$corresponds to the case that the loops of electrons and holes are completely overlapped in the $K$ valley as shown in Fig. 5(b) left.

When we increase $E$ from 0 to $E_{\text {cr }}^{+}$, since the intensity of LCP (RCP) increases (decreases), the $\eta$ rapidly increases. As we further increase $\tau E$ more than $\tau E_{\mathrm{cr}}^{+}$, the overlapping regions for LCP and RCP both decrease, which decreases each EL intensity and peak frequency. This makes that $\eta$ increases slower than the case of $\tau E<\tau E_{\mathrm{cr}}^{+}$. When the EL intensity of RCP vanishes at $\tau E=2.8 \times 10^{-7} \mathrm{~N} \mathrm{~s} \mathrm{C}^{-1}$ because of the vanishing overlapping region in the $K^{\prime}$ valley as shown in Fig. $6, \eta$ reaches to 1.0 . It is noted that the $E_{\mathrm{ph}, \sigma}^{\mathrm{m}}$ cannot be plotted in Fig. 7(a) when the corresponding intensity $I_{\sigma}^{\mathrm{m}}$ drops to zero in Fig. 7(b).

When the direction of in-plane electric field is exchanged, we should exchange the charge distributions of electron and hole at the same time (n-i-p junction) for keeping the forward bias. When we exchange direction of $\mathbf{E}$, as shown in Fig. 7, 


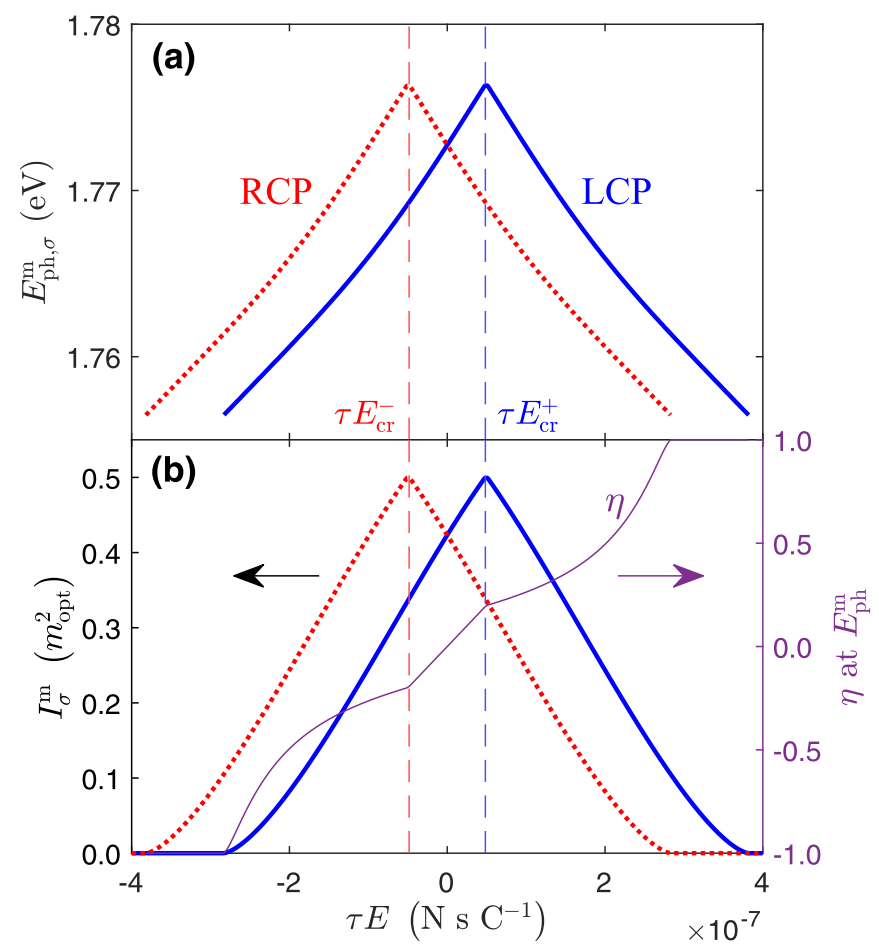

FIG. 7. (a) The EL photon energy at the maximum intensity of the EL spectra, $E_{\mathrm{ph}, \sigma}^{\mathrm{m}}$ for LCP and RCP as a function of $\tau E$. (b) The maximum intensity $I_{\sigma}^{\mathrm{m}}$ in units of $m_{\mathrm{opt}}^{2}$ and $\eta$ at $E_{\mathrm{ph}}^{\mathrm{m}}$ as a function of $\tau E . \tau E_{\mathrm{cr}}^{\sigma}$ is denoted by the vertical dashed line.

the emission of LCP and RCP exchanges which changes the sign of $\eta$. This situation designates the electrically tunable circularly polarized light source based on the valley polarization which is observed in the experiments [15,19-21].

\section{DISCUSSIONS}

In the present calculation, we assumed that the pseudo Fermi energy $E_{\mathrm{F}}$ for electrons and holes are taken to be the same. However, this assumption can be relaxed by giving the inequivalent electrochemical doping in the $\mathrm{p}$ and $\mathrm{n}$ regions. In this case, the electron and hole loops do not have the same area which gives the flat peak of the EL intensity. For example, if we consider a larger electron loop than a hole loop, RCP vanishes for a smaller $\tau E$ value than the case of Fig. 7 which is illustrated in Fig. 8. Thus it is an efficient way to get $\eta=1$ for relatively smaller $\tau E$ for EL of LCP. The corresponding strain can be small for the smaller area although the total EL intensity should be proportional to the area. It should be mentioned that the value of $\tau$ is not a constant but a function of energy of electrons or holes. Further, the value of $\mathbf{E}$ in

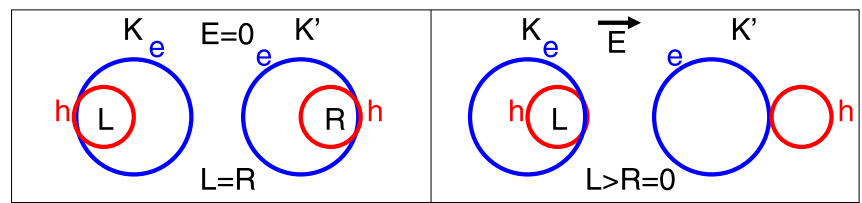

FIG. 8. If the hole loop is smaller than the electron loop, RCP vanishes for a smaller $\tau E$ than the case of same loops of hole and electron.

the $\mathrm{i}$ region depends on the width of the $\mathrm{i}$ region. Thus the estimation of $\tau E$ in the calculation and comparison with the experiment are necessary.

Another important point that we should address is the exciton effect. The binding energy of 2D exciton is given as a function of the number of carriers and the dielectric constants of the substrates. If the number of carriers is relatively large, the exciton formation in the i region is not expected. In fact, the experimental results show an evidence of a trion in the lower energy region of the EL spectra [15]. The equation of motion for the trion is beyond the scope of the present calculation.

The direction of strain is important for the present results. In the present case, the strain in the $y$ axis does not affect the pseudovector potential for $k_{y}$ in the zigzag nanoribbon $[37,55]$. Changing the strain in the present geometry gives strain dependent $\eta$ and the peak intensity (not shown) which should be compared with the experiment. On the other hand, the observation of the $\eta$ in the experiment can be used for estimation of the strain once the crystal orientation is given.

\section{CONCLUSIONS}

In this paper, we calculate EL intensity of the strained $\mathrm{MoS}_{2}$ in which the strain in TMD enhances the degree of circularly polarized EL in the p-i-n junction. Circularly polarized EL occurs because of (1) valley polarization, (2) the electron-hole asymmetry and (3) the in-plane electric field in the $i$ region. In an extreme condition, we get $100 \%$ LCP (RCP) emission in the EL without losing the EL intensity much. We hope that the theoretical prediction will be observed by the experiment.

\section{ACKNOWLEDGMENTS}

S.W. acknowledges the National Natural Science Foundation of China (Grant No. 11704165), the China Scholarship Council (Grant No. 201908320001), and the counterpart fund of Jinling Institute of Technology. M.S.U. and R.S. acknowledge JSPS KAKENHI (Grant No. JP18H01810).
[1] K. F. Mak, C. Lee, J. Hone, J. Shan, and T. F. Heinz, Phys. Rev. Lett. 105, 136805 (2010).

[2] A. Splendiani, L. Sun, Y. Zhang, T. Li, J. Kim, C.-Y. Chim, G. Galli, and F. Wang, Nano Lett. 10, 1271 (2010).

[3] A. Kuc, N. Zibouche, and T. Heine, Phys. Rev. B 83, 245213 (2011).
[4] W. Zhao, Z. Ghorannevis, L. Chu, M. Toh, C. Kloc, P.-H. Tan, and G. Eda, ACS Nano 7, 791 (2013).

[5] R. Roldán, J. A. Silva-Guillén, López-Sancho, M. Pilar, F. Guinea, E. Cappelluti, and P. Ordejón, Ann. Phys. 526, 347 (2014).

[6] C. Ruppert, O. B. Aslan, and T. F. Heinz, Nano Lett. 14, 6231 (2014). 
[7] S. Wang, C. Ren, H. Tian, J. Yu, and M. Sun, Phys. Chem. Chem. Phys. 20, 13394 (2018).

[8] S. Wang, H. Tian, C. Ren, J. Yu, and M. Sun, Sci. Rep. 8, 12009 (2018).

[9] D. Xiao, G.-B. Liu, W. Feng, X. Xu, and W. Yao, Phys. Rev. Lett. 108, 196802 (2012).

[10] J. Pu and T. Takenobu, Adv. Mater. 30, 1707627 (2018).

[11] C. R. Zhu, G. Wang, B. L. Liu, X. Marie, X. F. Qiao, X. Zhang, X. X. Wu, H. Fan, P. H. Tan, T. Amand, and B. Urbaszek, Phys. Rev. B 88, 121301(R) (2013).

[12] K. Ghalamkari, Y. Tatsumi, and R. Saito, J. Phys. Soc. Jpn. 87, 024710 (2018).

[13] S. Wang, F. R. Pratama, M. S. Ukhtary, and R. Saito, Phys. Rev. B 101, 081414(R) (2020).

[14] R. S. Sundaram, M. Engel, A. Lombardo, R. Krupke, A. C. Ferrari, P. Avouris, and M. Steiner, Nano Lett. 13, 1416 (2013).

[15] Y. J. Zhang, T. Oka, R. Suzuki, J. T. Ye, and Y. Iwasa, Science 344, 725 (2014).

[16] A. Kormányos, V. Zólyomi, N. D. Drummond, P. Rakyta, G. Burkard, and V. I. Fal'ko, Phys. Rev. B 88, 045416 (2013).

[17] S. Ono and K. Sugihara, J. Phys. Soc. Jpn. 24, 818 (1968).

[18] R. Saito, G. Dresselhaus, and M. S. Dresselhaus, Phys. Rev. B 61, 2981 (2000).

[19] M. Onga, Y. Zhang, R. Suzuki, and Y. Iwasa, Appl. Phys. Lett. 108, 073107 (2016)

[20] W. Yang, J. Shang, J. Wang, X. Shen, B. Cao, N. Peimyoo, C. Zou, Y. Chen, Y. Wang, C. Cong, W. Huang, and T. Yu, Nano Lett. 16, 1560 (2016).

[21] Y. Zhang, R. Suzuki, and Y. Iwasa, ACS Nano 11, 12583 (2017).

[22] A. Castellanos-Gomez, M. Poot, G. A. Steele, H. S. J. van der Zant, N. Agraït, and G. Rubio-Bollinger, Adv. Mater. 24, 772 (2012).

[23] T. Lorenz, J.-O. Joswig, and G. Seifert, 2D Mater. 1, 011007 (2014).

[24] R. K. Defo, S. Fang, S. N. Shirodkar, G. A. Tritsaris, A. Dimoulas, and E. Kaxiras, Phys. Rev. B 94, 155310 (2016).

[25] S. Bertolazzi, J. Brivio, and A. Kis, ACS Nano 5, 9703 (2011).

[26] P. Avouris, T. F. Heinz, and T. Low, 2D Materials: Properties and Devices (Cambridge University Press, Cambridge, 2017).

[27] X. Huang, Z. Zeng, and H. Zhang, Chem. Soc. Rev. 42, 1934 (2013).

[28] J. Munguía, G. Bremond, J. M. Bluet, J. M. Hartmann, and M. Mermoux, Appl. Phys. Lett. 93, 102101 (2008).

[29] L. Mennel, M. M. Furchi, S. Wachter, M. Paur, D. K. Polyushkin, and T. Mueller, Nat. Commun. 9, 516 (2018).

[30] R. Roldán, A. Castellanos-Gomez, E. Cappelluti, and F. Guinea, J. Phys.: Condens. Matter 27, 313201 (2015).

[31] W. S. Yun, S. W. Han, S. C. Hong, I. G. Kim, and J. D. Lee, Phys. Rev. B 85, 033305 (2012).

[32] J. Feng, X. Qian, C.-W. Huang, and J. Li, Nat. Photonics 6, 866 (2012).

[33] L. Wang, A. Kutana, and B. I. Yakobson, Ann. Phys. 526, L7 (2014).
[34] E. Scalise, M. Houssa, G. Pourtois, V. Afanas'ev, and A. Stesmans, Nano Res. 5, 43 (2012).

[35] P. Johari and V. B. Shenoy, ACS Nano 6, 5449 (2012).

[36] M. Ghorbani-Asl, S. Borini, A. Kuc, and T. Heine, Phys. Rev. B 87, 235434 (2013).

[37] H. Rostami, R. Roldán, E. Cappelluti, R. Asgari, and F. Guinea, Phys. Rev. B 92, 195402 (2015).

[38] S. Fang, R. Kuate Defo, S. N. Shirodkar, S. Lieu, G. A. Tritsaris, and E. Kaxiras, Phys. Rev. B 92, 205108 (2015).

[39] H. Rostami, A. G. Moghaddam, and R. Asgari, Phys. Rev. B 88 , 085440 (2013).

[40] See Supplemental Material at http://link.aps.org/supplemental/ 10.1103/PhysRevResearch.2.033340 for the derivation of the square of matrix element (Sec. S1) and EL in unstrained TMD (Sec. S2).

[41] A. F. J. Levi, Applied Quantum Mechanics (Cambridge University Press, New York, 2012).

[42] B. K. Ridley, Quantum Processes in Semiconductors (Oxford University Press, Oxford, 2013).

[43] M. Vila, N. T. Hung, S. Roche, and R. Saito, Phys. Rev. B 99, 161404(R) (2019).

[44] N. W. Ashcroft and N. D. Mermin, Solid State Physics (Saunders College Publishing, Orlando, 1976).

[45] U. K. Mishra and J. Singh, Semiconductor Device Physics and Design (Springer, Dordrecht, 2007).

[46] X. Wang and Z. M. Wang, Nanoscale Thermoelectrics (Springer, Cham, 2014)

[47] A. Ramasubramaniam, Phys. Rev. B 86, 115409 (2012).

[48] A. Grüneis, R. Saito, G. G. Samsonidze, T. Kimura, M. A. Pimenta, A. Jorio, A. G. Souza Filho, G. Dresselhaus, and M. S. Dresselhaus, Phys. Rev. B 67, 165402 (2003).

[49] H. Minden, IEEE Trans. Parts Mater. Packag. 1, 40 (1965).

[50] J. F. Nye, Physical Properties of Crystals: Their Representation by Tensors and Matrices (Clarendon Press, Oxford, 1985).

[51] M. H. Sadd, Elasticity: Theory, Applications, and Numerics (Academic Press, London, 2020).

[52] A. Kumar and P. K. Ahluwalia, Physica B 419, 66 (2013).

[53] K. Ghalamkari, Y. Tatsumi, and R. Saito, J. Phys. Soc. Jpn. 87, 063708 (2018).

[54] V. M. Pereira and A. H. Castro Neto, Phys. Rev. Lett. 103, 046801 (2009).

[55] K.-i. Sasaki and R. Saito, Prog. Theor. Phys. Suppl. 176, 253 (2008).

[56] K.-i. Sasaki, R. Saito, M. S. Dresselhaus, K. Wakabayashi, and T. Enoki, New J. Phys. 12, 103015 (2010).

[57] A. L. Kitt, V. M. Pereira, A. K. Swan, and B. B. Goldberg, Phys. Rev. B 85, 115432 (2012).

[58] A. L. Kitt, V. M. Pereira, A. K. Swan, and B. B. Goldberg, Phys. Rev. B 87, 159909(E) (2013).

[59] T. Fujita, M. B. A. Jalil, and S. G. Tan, Appl. Phys. Lett. 97, 043508 (2010).

[60] Z. Niu, J. Appl. Phys. 111, 103712 (2012).

[61] S.-K. Wang and J. Wang, Chin. Phys. B 24, 037202 (2015).

[62] A. J. Pearce, E. Mariani, and G. Burkard, Phys. Rev. B 94, 155416 (2016). 